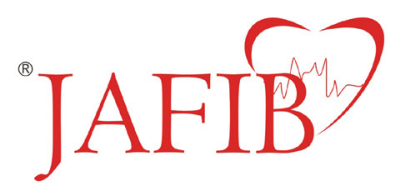

Original Research

Journal of Atrial Fibrillation

www. jafib.com

\title{
Percutaneous Vacuum-Assisted Thrombectomy Device Used for Removal of Large Vegetations on Infected Pacemaker and Defibrillator Leads as an Adjunct to Lead Extraction
}

\author{
Raymond H. M. Schaerf, Sasan Najibi, John Conrad \\ Providence St Joseph Medical Center Lead Management Center, Burbank, CA.
}

\begin{abstract}
This case series reports our early experience with a minimally invasive percutaneous method of safely removing large vegetations during lead extraction in septic cardiac implantable electronic devices (CIED). Debate exists concerning the management of vegetations involving these devices. Lead extraction is mandated for infections, but vegetations may embolize, causing complications. Surgical debridement is recommended; alternatives include cardiopulmonary bypass, minimally invasive thoracotomy, or transatrial approaches. The AngioVac device allows percutaneous right heart bypass and suction removal of vegetations under echocardiographic and fluoroscopic guidance. This case series describes our first 20 patients, all critically ill with persistent sepsis and vegetations despite long-term antibiotics. This series includes patients who would not have been eligible for alternative procedures due to contraindications and highlights the potential role of this new technology.
\end{abstract}

\section{Introduction}

Implantation of CIED, including implantable cardioverter defibrillators (ICDs), has increased in recent years due to results of large clinical trials and a corresponding expansion of indications for use. Historically, infection rates have been reported as ranging from $0.8 \%$ to $19.9 \%$ of patients. ${ }^{1}$ Mortality rates for infected CIEDs vary in the published literature, with highest rates occurring among patients treated with antibiotic alone (31\% to $66 \%)$ and as low as $13 \%$ to $33 \%$ with antibiotics and lead removal. ${ }^{2}$ In an 8 year review exploring increased utilization of these devices and resulting infections published in 2006, Voight and colleagues found a there was a $49 \%$ rise in the number of new cardiac rhythm management (CRM) devices over the study period. In the same period, the number of hospitalizations with CRM device infection increased 3.1-fold, the increase in infections has not been proportional to the increase in device utilization. ${ }^{3}$ The authors also found a CRM device infection conferred a significantly increased risk of in-hospital death (odds ratio $[\mathrm{OR}] 2.41, \mathrm{p}<0.001)$.

The Heart Rhythm Society consensus guidelines recommend

Key Words:

Lead Extraction, Percutaneous Thrombectomy, Vegetation.

Disclosures:

None.

Corresponding Author:

Raymond Schaerf,

Providence St Joseph Medical Center Lead Management Center,

2601 W. Alameda Avenue,

Burbank, CA 91505. these patients receive antibiotic therapy and removal of the infected hardware including generators and electrodes. ${ }^{4}$ Historically, patients with device-related infection and vegetations over $1 \mathrm{~cm}$ have often been referred for surgical thoracotomy for removal of the CRM system due to the risk of septic embolization. ${ }^{5}$ But many patients presenting with these larger vegetations may not eligible for an invasive procedure due to a myriad of factors including comorbidities, advanced age, debilitative previous surgeries; they may refuse the procedure.

This case series presents our early experience with a minimally invasive, percutaneous method facilitating removal of large vegetations $(2-6 \mathrm{~cm})$ during lead extraction in CIED patients with sepsis and vegetative endocarditis despite adequate antibiotic therapy.

Material and Methods

Device Background and Technique

The AngioVac cannula is a $22 \mathrm{Fr}$ stainless steel coil-reinforced venous drainage cannula designed to facilitate the removal of undesirable intravascular material via a balloon-actuated funnelshaped tip (see Figure 1). It is indicated for the removal of fresh or soft thrombi or emboli from vessels including, but not limited to, the superior and inferior vena cavae, iliofemoral veins, and right atrium. The removal of vegetations, and by extension the size limitations of vegetation which may be targeted for removal, is not specifically mentioned in the labeling. Two central venous access points are obtained, using any combination of internal jugular (IJ) and femoral veins. The AngioVac cannula is inserted through a $26 \mathrm{Fr}$ sheath and advanced over a 0.035 " stiff guidewire using fluoroscopic guidance; in 


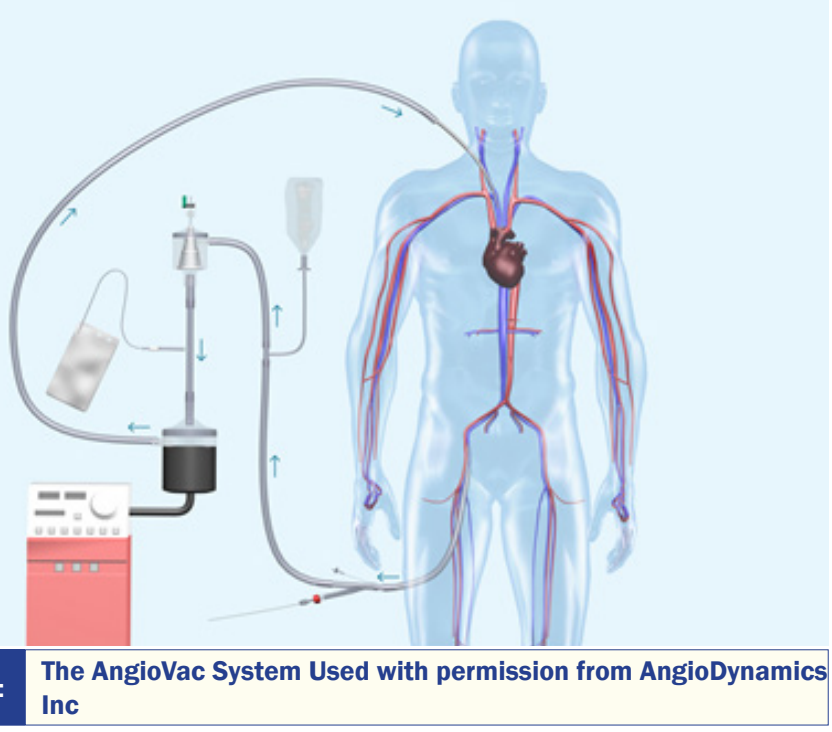

Figure 1:

cases involving the right atrium, transesophageal electrocardiography (TEE) and/or intracardiac ultrasound is utilized (see Figure 2).

Before engaging the material, flow through the cannula and circuit is initiated and optimized to a rate of 3 to $5 \mathrm{1} / \mathrm{min}$ or just prior to vascular collapse. Undesirable material is engaged by the tip of the cannula, and the shed blood is circulated through the extracorporeal circuit. Undesirable material is captured in an inline filter (See Figure 3). The shed, filtered blood is then reinfused to the patient via a $16 \mathrm{~F}$ or greater reinfusion cannula.

In most cases, the cannula is advanced to the material for direct removal of the material from the CIED leads. Once the vegetation is removed or debulked, the AngioVac cannula is used to follow the lead out of the right atrium and placed at the superior vena cava/ right atrium (SVC/RA) junction during removal of the lead to help protect against any remaining material present on the ICD leads from embolizing to the lungs. Our purpose is to examine the effectiveness of the AngioVac in the removal of large vegetations on infected pacemaker and defibrillator leads.

We perform our AngioVac procedure under general anesthesia with endotracheal intubation. Two large bore intravenous cannulae, an arterial line, and foley catheter are placed. A transesophageal echocardiogram (TEE) probe is placed and the patient is prepped from chin to knees. We secure venous access from both femoral veins,

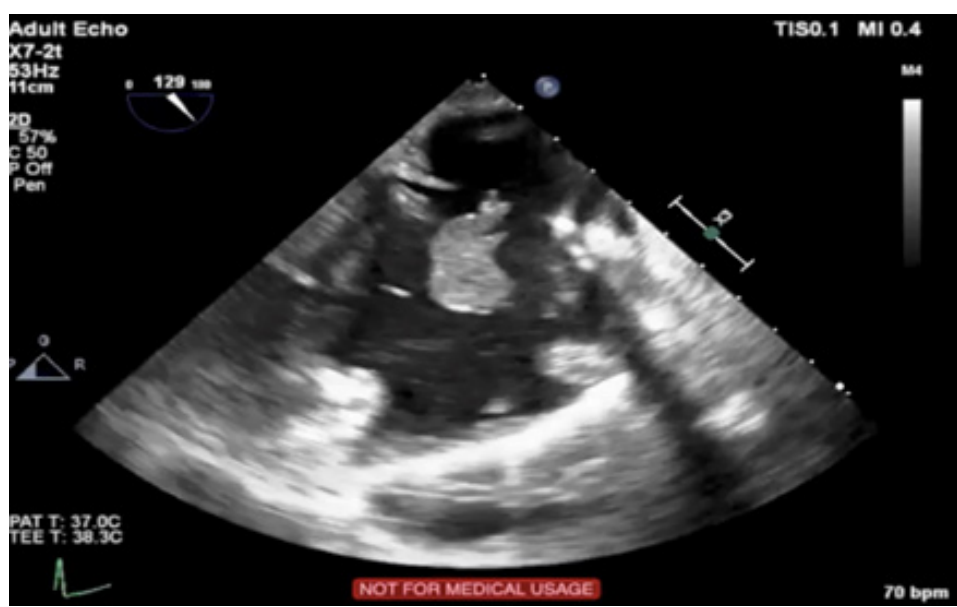

Figure 2:

Pre-procedure TEE showing vegetation on ventricular lead; blood culture positive for S. Aureus despite 6 weeks of antibiotic therapy

\begin{tabular}{|c|c|c|c|c|c|}
\hline Case & Age & Device(s) & $\begin{array}{l}\text { Veg Size } \\
(\mathrm{cm})\end{array}$ & $\begin{array}{l}\text { Length of } \\
\text { Stay (days) }\end{array}$ & Organism \\
\hline 1 & 82 & Biventricular ICD & 5 & 3 & Staphylococcus epidermidis \\
\hline 2 & 92 & DC Pacer & 6 & 3 & $\begin{array}{l}\text { Methicillin-sensitive } \\
\text { Staphylococcus aureus }\end{array}$ \\
\hline 3 & 78 & DC Pacer & 3 & 7 & Streptococcus bovis \\
\hline 4 & 87 & Biventricular ICD & 5 & 8 & MRSA \\
\hline 5 & 79 & DC Pacer & 3 & 3 & Staphylococcus epidermidis \\
\hline 6 & 71 & $\begin{array}{l}\text { DC Pacer and } 4 \\
\text { Leads }\end{array}$ & 5 & 3 & Staphylococcus epidermidis \\
\hline 7 & 89 & DC ICD & 2 & 2 & Staphylococcus capitis \\
\hline 8 & 59 & DC ICD & 3 & 3 & $\begin{array}{l}\text { Methicillin-sensitive } \\
\text { Staphylococcus aureus }\end{array}$ \\
\hline 9 & 82 & Biventricular ICD & 3 & 3 & Enterococcus \\
\hline 10 & 77 & $\begin{array}{l}\text { Subcutaneous } \\
\text { ICD }\end{array}$ & 2 & 4 & MRSA \\
\hline 11 & 86 & Biventricular ICD & 4 & 4 & MRSA \\
\hline 12 & 82 & DC Pacer & 3 & 3 & MRSA \\
\hline 13 & 48 & DC Pacer & 5 & 3 & Strep \\
\hline 14 & 65 & DC ICD & 4 & 9 & $\begin{array}{l}\text { Pseudomonas Staphylococcus } \\
\text { epidermidis }\end{array}$ \\
\hline 15 & 69 & DC ICD & 5 & 6 & $\begin{array}{l}\text { Coagulase-negative } \\
\text { staphylococci }\end{array}$ \\
\hline 16 & 74 & DC Pacer & 2 & 2 & $\begin{array}{l}\text { Methicillin-sensitive } \\
\text { Staphylococcus aureus }\end{array}$ \\
\hline 17 & 76 & Biventricular ICD & 2 & 2 & Staphylococcus hominis \\
\hline 18 & 82 & $\begin{array}{l}\text { Biventricular ICD } \\
\text { with } 2 \text { DF Leads }\end{array}$ & 3 & 4 & Streptococcus bovis \\
\hline 19 & 68 & $\begin{array}{l}\text { DC ICD with } 2 \text { DF } \\
\text { Leads }\end{array}$ & 3.5 & 3 & $\begin{array}{l}\text { Coagulase-negative } \\
\text { staphylococci }\end{array}$ \\
\hline 20 & 73 & Biventricular ICD & 4 & 4 & Staphylococcus epidermidis \\
\hline
\end{tabular}

and often have a guidewire placed in the right internal jugular vein.

Once the guidewires are in place, the patient is systemically heparinized and activated clotting time (ACT) is measured. We then place the Angiovac cannula into the right atrium under fluoroscopic guidance and TEE, and attain right heart bypass. Once right heart bypass is established, the extraction procedure is performed using either laser, mechanical, or traction alone, and vegetations are viewed under TEE. In our experience, the majority of the vegetations are moved when one or more culprit leads are freed by the extraction tools. When the procedure is completed, the heparin is reversed, and the cannulae are removed. Surgical debridement of the extraction

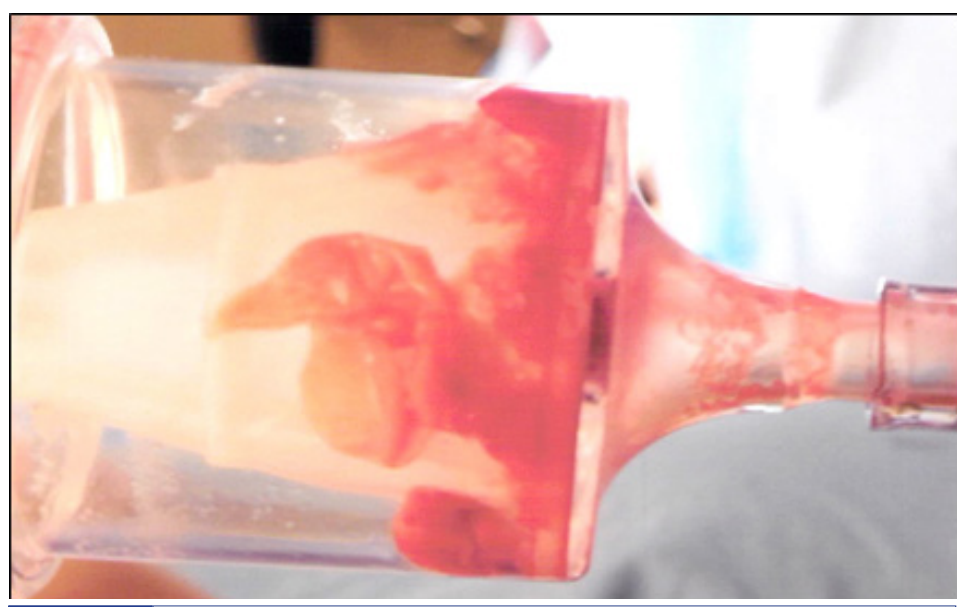

Figure 3: Large Vegetations visible in inline filter 
sites is then completed.

\section{Results}

All 20 patients survived the procedure and resolved their infections (Table 1: Patient Selection). The ACT values measured during the procedure all were within the range of 300-40 seconds. Two intraprocedural complications occurred. In Patient No. 4, an injury to an iliac vein occurred and was repaired with a stent. In another Patient No. 14, a pseudoaneurysm of a previous mycotic femoral aneurysm required resection and vein repair. With several exceptions, length of stay for patients was the same as our usual postoperative length of stay for infected patients. Our usual protocol is to discharge only after a definitive organism has been identified from the operative cultures. Patient No. 3 stayed an additional 3 days to have an unrelated procedure. Patient 4 was a Jehovah's Witness; the patient had preoperative and postoperative hemoglobin levels of 9 and $3 \mathrm{mg} / \mathrm{dl}$, contributing to an extended length of stay. Patient No. 15 presented with septic pulmonary emboli and required observation for antibiotics not covered by his insurance carrier for outpatient delivery. With the exception of Patient No. 15, none of the patients had positive postoperative blood cultures. Patient information was not gathered after hospital stay as part of a follow-up.

No clinical or ultrasound evidence of vegetative emboli was seen post-procedure. In our opinion, persistent endocarditis and presence of vegetations (despite adequate antibiotic therapy) with greater than or equal to $2 \mathrm{~cm}$, is an indication for this procedure. This is supported by similar findings where the utilization of the AngioVac assisted with the successful removal of larger, bulky vegetations. ${ }^{6,7}$ The procedure requires a team made up of surgeons (cardiothoracic and/or vascular) with specialty in cardiology, anesthesia, infectious disease, and perfusion.

\section{Conclusions}

Lead extraction in infected patients with vegetations has become a routine and safe procedure, done in many centers. We have found that even in relatively small sized vegetation cases, some patients have postoperative fevers and septic episodes which may be related to embolization of vegetation fragments, and may cause severe morbidity and death. We have found that the size of the vegetations is often underestimated by the preoperative transesophageal echocardiogram. Patients with documented vegetations who have positive blood cultures and persistent 2 to $3 \mathrm{~cm}$ or greater vegetations may benefit from a less invasive mechanical removal of their vegetations.

This single center case series describes our initial experience with a new minimally invasive approach to removal of right heart masses. It has replaced our previous method of sternotomy and cardiopulmonary bypass, which we felt would have been contraindicated in many of our patients in this series. A limitation of this study is the lack of follow-up data; further studies are necessary to reproduce the safe outcomes we have been fortunate to have obtained, but also to determine if this equipment might have more use than simply for 2 $\mathrm{cm}$ or larger vegetations. Furthermore, future studies examining the risks associated with breaking up larger vegetations before AngioVac utilization would shed more light on device efficacy.

\section{References}

1. Bluhm G. Pacemaker infections. A clinical study with special reference to prophylactic use of some isoxazolyl penicillins. Acta Med. Scand. Suppl. 1985;699:1-62.

2. Baddour Larry M, BettmannMichael A, BolgerAnn F, EpsteinAndrew
E, FerrieriPatricia, GerberMichael A, GewitzMichael H, JacobsAlice K, LevisonMatthew E, NewburgerJane W, PallaschThomas J, WilsonWalter R, BaltimoreRobert S, FalaceDonald A, ShulmanStanford T, TaniLloyd Y, TaubertKathryn A. Nonvalvular cardiovascular device-related infections. Circulation. 2003;108 (16):2015-31.

3. Voigt Andrew, ShalabyAlaa, SabaSamir. Rising rates of cardiac rhythm management device infections in the United States: 1996 through 2003. J. Am. Coll. Cardiol. 2006;48 (3):590-1.

4. Wilkoff Bruce L, LoveCharles J, ByrdCharles L, BongiorniMaria Grazia, CarrilloRoger G, CrossleyGeorge H, EpsteinLaurence M, FriedmanRichard A, KennergrenCharles E H, MitkowskiPrzemyslaw, SchaerfRaymond H M, WazniOussama M. Transvenous lead extraction: Heart Rhythm Society expert consensus on facilities, training, indications, and patient management: this document was endorsed by the American Heart Association (AHA). Heart Rhythm. 2009;6 (7):1085-104.

5. Grammes Jon A, SchulzeChristopher M, Al-BatainehMohammad, YesenoskyGeorge A, SaariChristine S, VrabelMichelle J, HorrowJay, ChowdhuryMashiul, FontaineJohn M, KutalekSteven P. Percutaneous pacemaker and implantable cardioverter-defibrillator lead extraction in 100 patients with intracardiac vegetations defined by transesophageal echocardiogram. J. Am. Coll. Cardiol. 2010;55 (9):886-94.

6. Divekar Abhay A, ScholzThomas, FernandezJoss D. Novel percutaneous transcatheter intervention for refractory active endocarditis as a bridge to surgeryangiovac aspiration system. Catheter Cardiovasc Interv. 2013;81 (6):1008-12.

7. Behrens George, BjarnasonHaraldur. Venous Thromboembolic Disease: The Use of the Aspiration Thrombectomy Device AngioVac. Semin Intervent Radiol. 2015;32 (4):374-8. 